Check for updates

Cite this: RSC Adv., 2017, 7, 41486

Received 27th July 2017

Accepted 16th August 2017

DOI: $10.1039 / c 7 r a 08306 h$

rsc.li/rsc-advances

\section{Tunable luminescence from two dimensional BCNO nanophosphor for high-contrast cellular imaging ${ }^{\dagger}$}

\author{
Bipin Kumar Gupta, (D) *a Pawan Kumar, ${ }^{\text {ab }}$ Garima Kedawat, ${ }^{c}$ Kanika, ${ }^{a}$ \\ Sajna Antony Vithayathil, ${ }^{d}$ Amit Kumar Gangwar, ${ }^{\text {ab }}$ Satbir Singh, ${ }^{\text {ab }}$ \\ Pradeep Kumar Kashyap, ${ }^{\text {ab }}$ Rimli Lahon, ${ }^{a}$ V. N. Singh, ${ }^{a}$ Abhay D. Deshmukh, (iD e \\ Tharangattu N. Narayanan, ${ }^{f}$ Nidhi Singh, ${ }^{\text {T Sarika Gupta }}{ }^{9}$ \\ and Benny Abraham Kaipparettu*dh
}

Rare-earth free and biocompatible two dimensional carbon based boron oxynitride (2D BCNO) nanophosphors were synthesized using facile auto-combustion of inexpensive compounds such as urea, boric acid and polyethylene glycol at ambient atmosphere and relatively low temperatures. The surface morphology and microstructure images indicate that the nanophosphor has 2D layered structures and analogous mixed hexagonal lattices of boron nitride (BN) and graphene (C). The nanophosphor exhibits a single, distinct and broad photoluminescence emission and this emission colour can be easily tuned from violet to deep red by varying the amount of boron/carbon content. The time-resolved and photoluminescence spectroscopic results indicate that B-O act as luminescence centers, which are responsible for the tunable luminescent properties while carbon impurities induce energy levels in the band gap of 2D BCNO nanophosphors. These tunable and biocompatible luminescent nanophosphors are used for in vitro high-contrast cellular imaging of HeLa cells derived from human cervical cancer cells as well as in vivo imaging in C57BL/6J mice. Hence, these novel multi-colour emitting nanophosphors provide a paradigm shift in rare-earth free biocompatible nanoprobes for next generation high-contrast in vitro and in vivo imaging applications.

\section{Introduction}

In depth studies on graphene in recent years have marked it as a very intriguing material with astonishing electronic properties, including high carrier mobility and very low resistivity, among others. ${ }^{1-3}$ However, though being an exquisite material,

${ }^{a}$ CSIR-National Physical Laboratory, Dr K S Krishnan Road, New Delhi, 110012, India. E-mail: bipinbhu@yahoo.com; Fax: +91-11-45609310; Tel: +91-11-45608284 ${ }^{b}$ Academy of Scientific and Innovative Research (AcSIR), CSIR-National Physical Laboratory Campus, Dr K S Krishnan Road, New Delhi 110012, India

${ }^{c}$ Department of Physics, Dyal Singh College, University of Delhi, New Delhi, 110003, India

${ }^{d}$ Department of Molecular and Human Genetics, Baylor College of Medicine, Houston, Texas 77030, USA. E-mail: kaippare@bcm.edu

${ }^{e}$ Energy Material and Devices Laboratory, Department of Physics, RTM Nagpur University, Nagpur, 440033, India

${ }^{f}$ TIFR-Centre for Interdisciplinary Sciences (TCIS), Tata Institute of Fundamental Research, Hyderabad - 500075, India

${ }^{g}$ National Institute of Immunology, Aruna Aseaf Ali Marg, J. N. U. Complex, New Delhi110067, India

${ }^{h}$ Dan L. Duncan Cancer Center, Baylor College of Medicine, Houston, TX 77030, USA $\dagger$ Electronic supplementary information (ESI) available. See DOI: 10.1039/c7ra08306h graphene possesses a disadvantage of being sensitive to its ambient surroundings, so much so that its properties can be wrecked by other materials present in close proximity. For a system to be able to mimic graphene and yet not allow alterations in its electronic properties by the environment, the material particularly must have a large electronic band gap and should be free from dangling bonds. Another material, well exploited due to its isoelectric resemblance with graphene is hexagonal boron nitride (h-BN). ${ }^{4}$ The h-BN, a well-known conventional and non-toxic material has been used to provide better electrical insulation, high heat resistance and good lubricant properties. ${ }^{5}$ The high corrosion resistance, high thermal conductivity, thermal stability under even oxygen atmosphere make h-BN a unique among other 2D members. ${ }^{6,7}$ This ultra-thin insulation atomic layer has many applications in electronic industry.

Tuning the physical properties by hybridization of different materials is one of the rapidly growing advancements in material science at present scenario. Due to the uncanny resemblance of h-BN with other graphene analogue nanostructures, its alternating nitrogen and boron atoms arrangement can bear addition of carbon. Boron carbon nitrate (BCN) is such a system where the introduction of $\mathrm{BN}$ into graphene or vice versa 
can change the properties of individual systems. Various approaches have been used to achieve this hybrid system. ${ }^{7-11}$ The in-plane doping of BN in atomic layer of graphene was reported. ${ }^{7}$ Further, the recent advances made by many groups display the modification in the light emission and $\mathrm{H}_{2}$ sorption properties of $(\mathrm{B}, \mathrm{N})$ rich $(\mathrm{BCN})$ with control over carbon and oxygen content. ${ }^{12-14}$ Since, BCN compounds also offer visible light emission, these can be considered as metal-free phosphors without any appreciable toxicity. ${ }^{12}$ Various inorganic phosphors have been developed over the years. Generally they require a phosphor to be chemically inert and thermally stable to achieve high luminescence efficiency with wide range of excitation and emission wavelengths. ${ }^{15-17}$ Oxide, oxysulphide, oxynitride and nitride compounds have been found to possess such properties and considered as the host lattices for rare earth (RE)-ions phosphors. ${ }^{14}$ As activators, RE-ions provide desired characteristics in a phosphor material. ${ }^{\mathbf{1 8 , 1 9}}$ However, its utility is limited because it is very expensive in its any form such as chlorides, acetates and oxides. Moreover, its synthesis procedure is quite cumbersome due to requirement of high temperature and pressure conditions. Hence, the usage of RE-ions phosphors is delimited in the future due to its lack of economically unfeasible and environmentally harmful characters. Therefore, the phosphor based on rare-earth free materials is in high demand because it is less expensive, comparatively non-toxic, thermochemically stable and eco-friendly in nature. Moreover, they possess high luminescence efficiency with broad excitation and emission spectrum. Importantly, boron carbon oxynitride (BCNO) can address several drawbacks of RE-ions phosphors with an advantage of tunable photoluminescent behavior. ${ }^{\text {20-24 }}$ With its composition, BCNO compounds are expected to exhibit wide electronic properties from insulating to conductor. ${ }^{11}$ Recently, few works on the synthesis of BCNO phosphors by thermal catalytic CVD, solid-state reactions, microwave plasma CVD and one-step liquid process have been reported including its application in the white light emitting diodes (WLEDs). ${ }^{\mathbf{4 1 1 , 2 5}}$ In addition, hydrogen storage, electronic devices and electron field-emission are some of the other attractive applications of BCNO. ${ }^{15-17}$ Though, BCNO offers a wide range of advantages over phosphor, currently it is challenging to obtain the high purity BCNO nanomaterials. To the best of our knowledge, there have been limited reports on the synthesis of BCNO and its application in the optoelectronic devices. However, so far, no studies have been reported on the use of BCNO as nanobioprobes for bioimaging applications. Previously, we have reported the yellow colour emitting 2D BCNO nanophosphor for WLEDs applications. ${ }^{4}$ Now, we are exploring the details of the synthesis of colour tunable 2D BCNO nanophosphor for in vitro and in vivo high-contrast cellular imaging.

The BCNO is reported as a highly efficient and environment friendly phosphor with its emission ranging from whole visible region to near UV region. Thus, with high emission yield and tunable emission for desired wavelength, BCNO paves the way for a broad range of applications. Since, its prospective biological applications being one of the most unexplored areas, we undertook this study to probe into the possibilities of using
BCNO as a biocompatible nanoprobe for high contrast cellular imaging. The theoretical calculations ${ }^{\mathbf{1 4 , 2 2}}$ and experimental evidence $^{8-10}$ have indicated that tunable visible emission and high quantum yield can be achieved in BCNO compounds with metal-free phosphors and low toxicity property.

Since the luminescent materials have been proposed as potential materials for detection and manipulation of biological systems, most of the studies are directed towards their biocompatibility and cytotoxicity which, mostly depends directly on the particle size and shape of the materials. A few studies on the synthesis of size-controlled europium doped yttrium oxide nanophosphor with small particle size (in nanometer range) have been reported. ${ }^{18}$ However, it is well-known that for biolabelling or any sort of biological applications, the particle size must be well below $10 \mathrm{~nm}$. Larger particle size can adversely affect the biological system and intracellular tracking during its transport through vascular endothelium and excretory process through urine.

Herein, we report the synthesis of $2 \mathrm{D}$ BCNO nanophosphor using auto-combustion method ${ }^{4}$ based on the reaction of urea, boric acid and polyethylene glycol. The emission colour clearly depends on the variation of $\mathrm{B} / \mathrm{C}$ mmolar ratio and this rareearth free tunable visible colour emitting biocompatible 2D BCNO nanophosphors are demonstrated to be a potential bionanoprobe for in vitro and in vivo high-contrast cellular imaging applications.

\section{Experimental}

\subsection{Synthesis of 2D BCNO nanophosphors}

The 2D BCNO nanophosphor was prepared using customized auto-combustion method in which boric acid $\left(\mathrm{H}_{3} \mathrm{BO}_{3}\right.$, boron source), urea or carbamide $\left(\mathrm{CO}\left(\mathrm{NH}_{2}\right)_{2}\right.$, nitrogen source) and polyethylene glycol(carbon source, PEG, $\left.M_{\mathrm{W}}-10000\right)$ were used as the starting materials. As we have previously reported, PEG is one of the appropriate carbon source materials for obtaining best PL intensity. ${ }^{4}$ These materials were mixed in $120 \mathrm{gm}$ of ultrapure water and stirred vigorously at $100{ }^{\circ} \mathrm{C}$ for obtaining a clear transparent aqueous solution. Unless otherwise stated, the quantity of the boric acid and urea in the precursor solution were fixed at $0.025 \mathrm{~mol}$ and $0.25 \mathrm{~mol}$, respectively and the mole amount of PEG were changed from 0 to $0.35 \mathrm{~mol}$. The resulted solution was heated in closed box type furnace to $400{ }^{\circ} \mathrm{C}$ at the rate of $6{ }^{\circ} \mathrm{C} \mathrm{min}^{-1}$ and kept at this temperature for $10 \mathrm{~min}$ to form pyrolsate (fluffy dry-gel powder form). The pyrolsate step is important in which organic compound is pyrolyzed under condition that would not allow organic compounds to vanish by combustion, thus, the $\mathrm{C} / \mathrm{N}$ ratio remains constant. In order to have uniform carbon composition, the pulverized pyrolsate mixture was disintegrated with the help of furnace. This mixture was transferred into a ceramic crucible and heated to $800{ }^{\circ} \mathrm{C}$ in box furnace at the rate of $25^{\circ} \mathrm{C} \mathrm{min}{ }^{-1}$ for $10 \mathrm{~min}$ to obtain BCNO precursor. The complex chemical changes may take place in the mixture with this optimized temperature. For achieving homogeneity throughout the mass, the as-prepared precursor was subsequently annealed at $750{ }^{\circ} \mathrm{C}$ for $45 \mathrm{~min}$ under ambient conditions. Here, in this process, the $\mathrm{B} / \mathrm{N}$ ratio 
(0.1) was kept constant, whereas B/C ratio was varied from 25 to 71.42 in order to control the emission spectra of the obtained products, as shown in Table T3 (see ESI $\dagger$ ). The 2D BCNO nanophosphor samples having different $\mathrm{B} / \mathrm{C}$ ratio in $\mathrm{mmol}$ units is labelled as $\mathrm{A}(\mathrm{B} / \mathrm{C}=25 / 0), \mathrm{B}(\mathrm{B} / \mathrm{C}=25 / 0.05), \mathrm{C}(\mathrm{B} / \mathrm{C}=25$ / $0.10), \mathrm{D}(\mathrm{B} / \mathrm{C}=25 / 0.15), \mathrm{E}(\mathrm{B} / \mathrm{C}=25 / 0.20), \mathrm{F}(\mathrm{B} / \mathrm{C}=25 / 0.25), \mathrm{G}$ $(B / C=25 / 0.30)$ and $\mathrm{H}(\mathrm{B} / \mathrm{C}=25 / 0.35)$. The oxygen content varies within a very limited range for these samples. The oxygen atoms were found to be present in the form of boron oxide, and boron oxynitride that can be considered as an interfacial zone bridging regions of boron nitride and the residual boron oxide. It is quite evident that the emission color wavelength can be easily tuned from violet to deep red regions simply by varying the $\mathrm{B} / \mathrm{C}$ ratio. This is further explained in photoluminescence section with more clarity.

\subsection{Characterization}

The phase purity identification, gross structural characterization and the bonding state of the as-obtained product were analyzed by XRD technique (Rigaku: MiniFlex, $\mathrm{CuK} \alpha_{1} ; \lambda=$ $1.5406 \AA$ ). The slow X-ray scan was performed in the $2 \theta$ range from 10 to $80 \mathrm{deg}$. with a scan step of $0.01 \mathrm{deg}$. Prior to the XRD measurement, the diffractometer was calibrated using silicon powder as reference material $\left(d_{111}=3.1353 \AA\right) .{ }^{26}$ The accurate lattice parameters were also obtained through a least square fitting method using computer-based unit cell refinement software. The chemical bonding status was examined with Fourier transformation infrared (FTIR) spectroscopy by NICOLET 6700 instrument using $\mathrm{KBr}$ pellets. The thickness of the $2 \mathrm{D}$ BCNO layer was measured by atomic force microscope (AFM, Model no. NT-MDT Solver Scanning probe Microscope). The surface morphology and micro-structural characterization were performed by scanning electron microscopy (SEM, Model no. EVO MA 10 VPSEM) and high resolution transmission electron microscopy (HRTEM, Model no. Technai G20-twin, $200 \mathrm{kV}$ with super twin lenses having point and line resolution of $0.144 \mathrm{~nm}$ and $0.232 \mathrm{~nm}$, respectively) equipped with energy dispersive $\mathrm{X}$ ray analysis (EDAX) facilities for elemental studies. The 2D BCNO nanophosphor chemical composition was analyzed by an electron energy-loss spectrometer (EELS) using a spectrometer attached to the transmission electron microscope. EDAX mapping of the 2D BCNO nanophosphor has been performed by using field scanning emission microscope (FESEM) Carl ZEISS-SUPRA 40 VP equipped with EDAX facility. UV-visible spectra were measured by a high resolution UV-vis spectrophotometer (MODEL no. LS 55). The photoluminescence (PL) spectra of nanophosphors were carried out using photoluminescence spectrometer (Edinburgh, FLSP-920), where Xenon flash lamp acts as source of excitation. In order to measure the absolute quantum efficiency of 2D BCNO nanophosphors, we used an integrating sphere, which is equipped with Edinburgh, FLSP-920 spectrometer. The estimation of quantum yield was estimated on the integral fraction of luminous flux and radiant flux as per well-known standard method. Time-resolved spectroscopy was performed by photoluminescence spectrometer with EPL $375 \mathrm{~nm}$ picoseconds pulsed diode laser as a source of excitation. The CIE color coordinates were measured using colorimeter C1210, serial no. 1296104. All the optical photographs were taken from the digital CANON Power Shot SX600 HS $(16 \times$ megapixels, $5 \times$ optical zoom) camera.

\subsection{Biocompatibility}

The HeLa cells were cultured and maintained in DMEM high glucose medium (Invitrogen) containing $4.5 \mathrm{~g} \mathrm{~L}^{-1}$ D-glucose, $4 \mathrm{mM}$ L-glutamine, and $110 \mathrm{mg} \mathrm{L}^{-1}$ sodium pyruvate, supplemented with $10 \%$ fetal bovine serum (FBS), $100 \mathrm{IU} \mathrm{mL}^{-1}$ penicillin and $100 \mu \mathrm{g} \mathrm{mL}^{-1}$ streptomycin in a humidified incubator at $37^{\circ} \mathrm{C}$ with $5 \% \mathrm{CO}_{2}$. About $\left(4 \times 10^{3}\right.$ cells $)$ were plated in each wells of a 96 well cell culture plate with $100 \mu \mathrm{L}$ of culture medium. After overnight incubation, anticancer drug or nanophosphor B, D, E and G diluted in cell culture medium were added to each wells in different concentration ranging from (0$100 \mu \mathrm{g} \mathrm{mL}^{-1}$ ) in triplicate. After $24 \mathrm{~h}$ and $48 \mathrm{~h}$ of incubation, the medium containing nanophosphors were removed, and the cells were washed gently with $500 \mu \mathrm{L}$ warm, sterile phosphate buffer solution (PBS). MTT (3-(4,5-dimethyl-2-yl)-2,5-diphenyltetrazolium bromide) assay was performed as described previously $^{27}$ using a Tecan Infinite M200 microplate reader (Mannedorf, Switzerland).

\subsection{In vitro bioimaging}

The cells were cultured and maintained in DMEM medium as described above. For bioimaging, $1 \times 10^{4}$ cells were plated in each well of a 4 well sterile chamber slides (Nunc, USA) with 500 $\mu \mathrm{L}$ culture medium. After overnight culture, $25 \mu \mathrm{g} \mathrm{mL} \mathrm{m}^{-1}$ of samples B, D, E, and G were added to the culture medium. After overnight incubation, medium was removed from the cells and was washed two times with $1 \mathrm{~mL}$ PBS. Cells were fixed using $1 \%$ paraformaldehyde and mounted with Vecta shield antifade mounting media with or without 4'-6-diamidino-2-phenylindole (DAPI, Vector Laboratories, Inc., CA). A Zeiss LSM 510 META confocal microscopy (Carl Zeiss) equipped with CApochromat40X/1.2W Corr and plan-Apochromat 63X/1.4 N.A. oil immersion objectives was used for bioimaging. The images were analyzed by the Zeiss LSM 510 software.

\subsection{In vivo imaging studies}

Whole body imaging was performed using Kodak in vivo FX PRO (Kodak, Rochester, NY, USA) employing Care stream Molecular Imaging software version 5.0.2.3.0 (Care stream Health Inc., Rochester, NY, USA). Six weeks old, C57BL/6J mice were used. The control and BCNO red and blue nanophosphors injected in C57BL/6J mice were anesthetized with a combination of xylazine (10 $\mathrm{mg} \mathrm{kg}^{-1}$ ) and ketamine (75 $\mathrm{mg} \mathrm{kg} \mathrm{kg}^{-1}$ ) administered intraperitoneally. Following anesthesia, mice were injected with BCNO red and blue nanophosphors intraperitoneally and images were acquired at different time intervals such as $15 \mathrm{~min}$, $1 \mathrm{~h}$ and $2 \mathrm{~h}$. Fluorescence images of whole body along with their controls were acquired in multi-wavelength mode using the following settings: exposure type - standard, exposure time - 
5 min, excitation filter $-410 \mathrm{~nm}$, emission filter $-600 \mathrm{~nm}$ (red) and $455 \mathrm{~nm}$ (blue) f-stop - 4.11, FOV - $160 \mathrm{~mm}$.

2.5.1 Animal ethics statement. All mice experiments were approved by animal ethics committee of National Institute of Immunology (IAEC no. 276/11) and conducted in accordance with horizontal legislation on the protection of animals used for scientific purposes, EU directive 2010/63/EU.

\section{Results and discussion}

2D BCNO nanophosphor has been synthesized using customized auto-combustion method. The structural purities of 2D BCNO nanophosphor were studied by powder X-ray diffraction. Fig. S1† shows the XRD patterns of synthesized samples A, B, C,
D, E, F, G and H. Ah-BN peak at $26.7^{\circ}$; JCPDS no. $75-1681$ are present in all powder samples. ${ }^{4}$ These extraneous peaks are consequence of unreacted boric acid, as shown in our previous report. ${ }^{4}$ The graphene-facilitated formation of h-BN has been observed and these overlapped signals indicate the hexagonal phase of 2D BCNO nanophosphor with lattice parameters, $a=$ $0.248 \mathrm{~nm}, b=0.248 \mathrm{~nm}$ and $c=0.663 \mathrm{~nm}$. Fig. 1a exhibits the repeated pattern of proposed unit cell of $2 \mathrm{D}$ BCNO nanophosphor on the basis of experimentally observed FTIR, EELS, AFM and TEM/HRTEM. Here, boron atoms are surrounded by three nitrogen atoms; out of which, two nitrogen atoms was replaced by a carbon atom and an oxygen atom respectively to form unit cell of BCNO. In hexagonal-boron nitride (h-BN) lattice, carbon and oxygen atoms act as impurity atoms. A
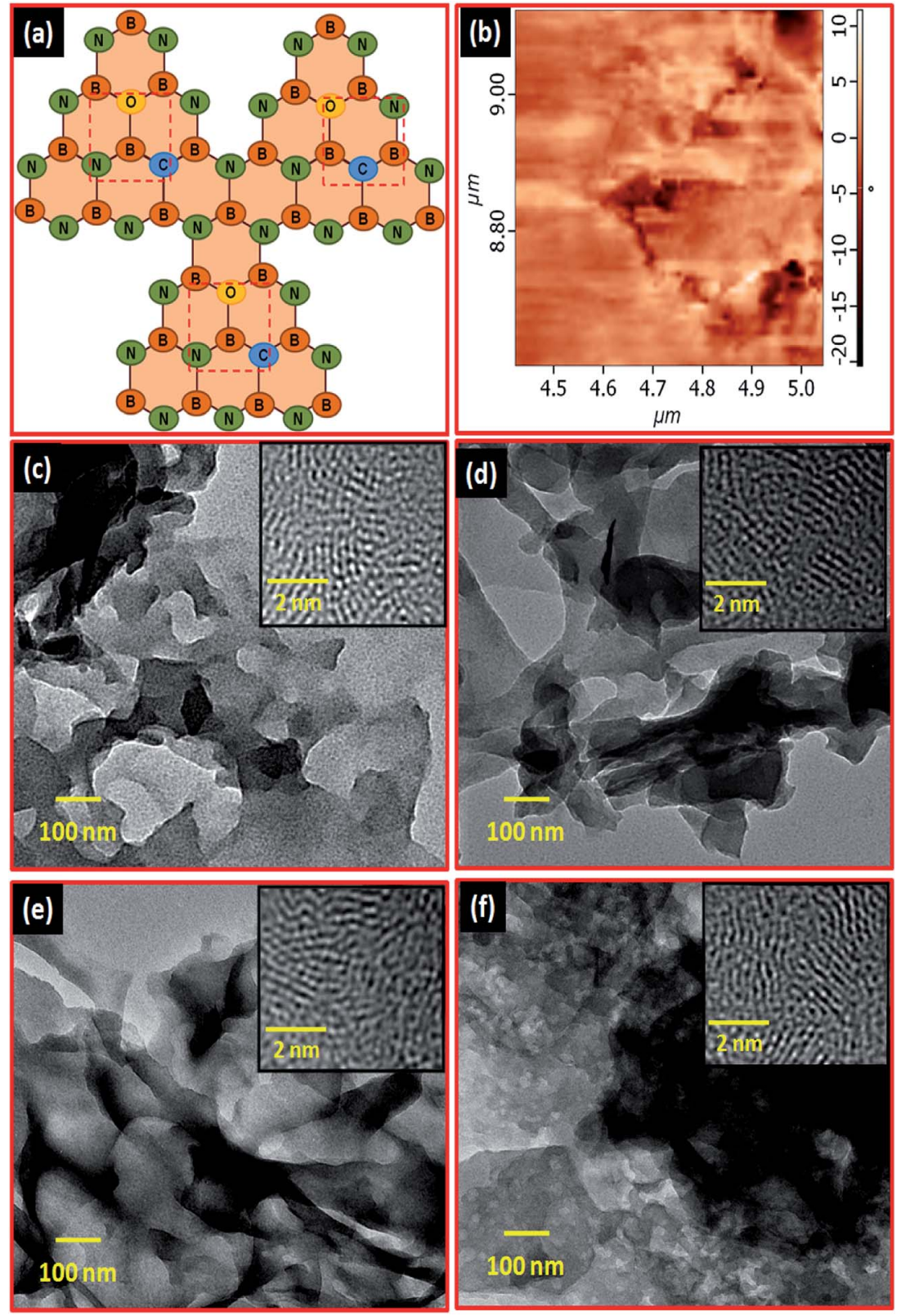

Fig. 1 Proposed unit cell and microstructural features of 2D BCNO nanophosphor: (a) schematic drawing of the repeated pattern of proposed unit cell of 2D BCNO nanophosphor (b) AFM micrograph of the 2D BCNO nanophosphor, TEM images of (c) B, (d) D, (e) E and (f) G samples. 
typical FTIR spectrum in $600-2000 \mathrm{~cm}^{-1}$ range for sample B is shown in Fig. S2 (see ESI $\dagger$ ). From the IR transmittance spectrum, the presence of BCNO was evident by $\mathrm{B}-\mathrm{N}, \mathrm{B}-\mathrm{C}, \mathrm{N}-\mathrm{B}-\mathrm{O}$ and $\mathrm{B}-\mathrm{N}-\mathrm{B}$ stretching vibrations modes at $\sim 1418,1072,942$, and $711 \mathrm{~cm}^{-1}$, respectively. ${ }^{4}$ The presence of $\mathrm{B}-\mathrm{N}$ linkage (stretching mode, $\sim 1418 \mathrm{~cm}^{-1}$ ) are attributed to in-plane and out-of-plane vibration modes of the $\mathrm{sp}^{2}$ hybridized $\mathrm{BN}^{28,29}$ The FTIR spectra of B, D, E and G samples having different amount of carbon content are shown in inset of Fig. S2 (see ESI $\dagger$ ). Moreover, introduction of carbon in the system does not result in any observable change in the shape of the IR spectra, indicating that the carbon amount in the as-formed BCNO nanophosphor may be very low. Thus, from the FTIR results, the formation mechanism of BCNO nanophosphor is confirmed. Further, the AFM technique affirmed the quality of the $2 \mathrm{D}$ BCNO layer. AFM image of the as-synthesized 2D BCNO nanophosphor of sample $\mathrm{G}$ is shown in Fig. $1 \mathrm{~b}$ reveals that ultrathin sheet-like nanostructure assembling its 2D structure. To determine the irregularity and width of layers line-scan profile was taken. The resultant line-scan profile specifies a thickness of 2D BCNO nanophosphor layer $\sim 2 \mathrm{~nm}$ is shown in Fig. $\mathrm{S} 3$ (see ESI $\dagger$ ).

The BCNO nanophosphor chemical composition was further examined via an electron energy-loss spectrometer (EELS). The EELS spectrum of sample B, D, E and G are shown in Fig. S4. $\dagger$

Further investigations were carried out to effectively enhance the performance of as-synthesized 2D BCNO nanophosphors derived from PEG as a carbon source. ${ }^{4}$ The surface morphology and chemical state of $\mathrm{B}, \mathrm{C}, \mathrm{N}$ and $\mathrm{O}$ elements were analyzed using SEM, TEM, EDAX and EDAX mapping. Fig. S5† represent SEM images of samples B, D, E and G, respectively. The SEM results of these nanophosphors show that ultrathin $2 \mathrm{D}$ randomly oriented layered nanostructure of nanophosphor. ${ }^{4}$ Fig. S6 $†$ represent EDAX spectrum of the 2D BCNO nanophosphor of sample B, sample D, sample E and sample G, the area from where EDAX spectrum was taken marked by the plus symbol in inset of Fig. S6. $\uparrow$ The elements detected by EDAX are largely comprised of $\mathrm{B}, \mathrm{C}, \mathrm{N}$, and $\mathrm{O}$. This result was consistent with the composition of the $2 \mathrm{D}$ BCNO nanophosphor. The presence of gold element is also observed due to the gold coating on pallet of BCNO nanophosphor prior to EDAX analysis. Fig. S7† shows the EDAX mapping of the BCNO nanophosphor which clearly shows the presence of $\mathrm{B}, \mathrm{C}, \mathrm{N}$ and $\mathrm{O}$ in the 2D BCNO layered nanophosphor. TEM images of samples B, D, E and $G$ are shown in Fig. 1c-f. It is clear from the figure that the samples are layered in nature and composed of various nanosheets. The high-resolution TEM (HRTEM) images of samples B, D, E and G are also given in inset of the Fig. 1c-f, respectively. These images clearly reveal lattice fringes with an interplanar distance of $0.34 \mathrm{~nm}$. The 2D BCNO nanophosphor can also be confirmed by selected area electron diffraction (SAED) pattern of samples B, D, E and G, as shown in Fig. S8 (see $\mathrm{ESI} \dagger)$, respectively. The weak diffraction rings are due to the stacking of few layers, which implies that, the prepared phosphors contain the crystals of hexagonal boron nitride (h-BN, JCPDS no. 73-2095). The EDAX spectrum of samples B, D, E and $\mathrm{G}$ are shown in Fig. S9-S12 (see ESI $\dagger$ ), respectively. The corresponding energy dispersive X-ray spectroscopy (EDAX) results reveal that microstructure consist of $\mathrm{B}, \mathrm{C}, \mathrm{N}$, and $\mathrm{O}$ elements, indicating the formation of BCNO nanophosphor. These results are consistent with previously reported results on BCNO nanophosphor. ${ }^{4}$

Fig. 2a shows the UV-vis absorption spectra of as-synthesized samples A, B, C, D, E, F, G and $\mathrm{H}$ (in water medium) in the range of 250-1000 nm. A shoulder peak centered at $295 \mathrm{~nm}$ appears in absorption spectra, which is induced by the transition between carbon impurity levels. The absorbance increases with increasing the carbon content in BCNO nanophosphor and broad absorption spectra in the range 300-600 nm may be assigned to the absorption of carbon related defects levels in BCNO nanophosphor. The normalized PL spectra of 2D BCNO nanophosphor having different compositions (B/C ratio) are shown in Fig. 2b. Each powder sample (A, B, C, D, E, F, G and H) was excited at different excitation wavelengths (sample A-315 nm, sample B-370 nm, sample C-385 nm, sample D-415 nm, sample E-470 nm, sample F-478 nm, sample G$485 \mathrm{~nm}$ and sample $\mathrm{H}-510 \mathrm{~nm}$ excitation wavelengths) as shown in Fig. $2 \mathrm{~b}$ and Table T1. $\dagger$
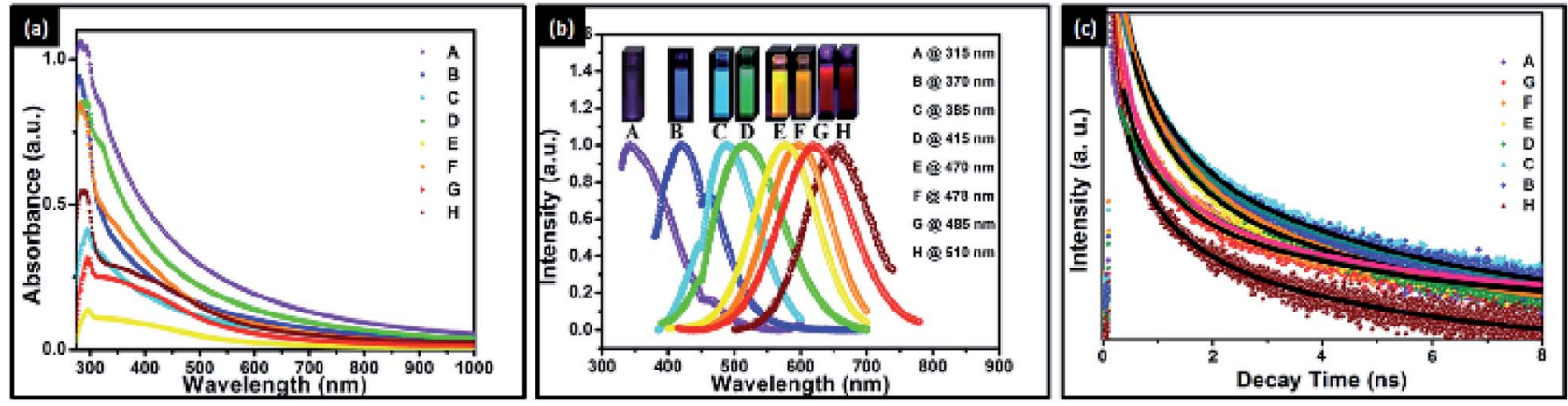

Fig. 2 Spectroscopic analysis of BCNO nanophosphor: (a) UV-vis absorption spectra of as-synthesized A, B, C, D, E, F, G and H samples, (b) PL emission spectra of $2 \mathrm{D} B C N O$ nanophosphor samples prepared with various $B / C$ molar ratios $(A=25 / 0, B=25 / 0.05, C=25 / 0.10, D=25 / 0.15, E$ $=25 / 0.20, F=25 / 0.25, G=25 / 0.3$ and $H=25 / 0.35$ ) and the inset exhibits multi-colour emissions from violet to deep red under excitation of $370 \mathrm{~nm}$ UV lamp for samples B-H in water medium, except sample A excited with $254 \mathrm{~nm}$ UV lamp, (c) time-resolved photoluminescence decay spectra of as-synthesized A, B, C, D, E, F, G and $\mathrm{H}$ samples with fitting curve. 
The multi-colour emissions from violet to deep red under excitation of $370 \mathrm{~nm}$ UV lamp for samples B-H in water medium, except sample A excited with $254 \mathrm{~nm} \mathrm{UV} \mathrm{lamp} \mathrm{are}$ shown in inset of Fig. 2b. Samples A-H under room light in water medium is shown in Fig. S13. $\dagger$ The PL emission of $2 \mathrm{D}$ $\mathrm{BCNO}$ nanophosphor (fixing the $\mathrm{B} / \mathrm{N}$ ratio and tailoring the $\mathrm{B} / \mathrm{C}$ ratio) is tailored according to the concentrations of $\mathrm{B} / \mathrm{C}$ ratio value ranging from $25 / 0$ to $25 / 0.35$ and subsequently leading to single, intense and different colour emitting (violet to deep red) from BCNO nanophosphor. Carbon atoms are crucial for controlling the emission band of 2D BCNO nanophosphor that leads to the changes in emission colour of BCNO nanophosphor. ${ }^{4}$ The carbon content introduced from the PEG material within the BCNO nanophosphors changed the chemical composition. The carbon vacancies substitute or intercalate in the crystal lattice of h-BN. This result in variations in the BCNO band gap and caused shifts in the PL peaks. ${ }^{11}$ Previous studies have reported that the band gaps of $\mathrm{BCN}$ compounds depend strongly on the atomic arrangement within the compounds., ${ }^{\mathbf{4 1 1}}$ The results described above suggest that the combination of a low synthesis temperature and a high PEG fraction leads to red-shifting of the BCNO phosphors. The comprehensive effects of $\mathrm{B} / \mathrm{C}$ molar ratio on the peak wavelength of the BCNO nanophosphors can be clearly seen in Fig. 2b. With the decrease in the B/C molar ratio, the peak position shifts towards longer wavelength. As a result, we succeeded in developing a rare earth free BCNO nanophosphors with tunable emission in the range from 342 to $654 \mathrm{~nm}$. The proposed energy level diagram of multi-colour emission is shown in Fig. S14. $\dagger$

Furthermore, these multi-colour emitting BCNO nanophosphors with constituent atoms $\mathrm{B}, \mathrm{C}, \mathrm{N}$ and $\mathrm{O}$ may have different diffusion rates to the proper lattice positions creating some defects in the structure. These defect levels are widely distributed in the electronic band structure of BCNO, which is considered to be the main reason for the broadened PL spectra. These results reveal that the resultant 2D BCNO nanophosphors emit all the visible range colours from violet to deep red. The chromaticity analysis was performed to further investigate the PL properties of the 2D BCNO nanophosphors. The chromaticity co-ordinate $(x, y)$ of the $2 \mathrm{D}$ BCNO nanophosphors were prepared with different $\mathrm{B} / \mathrm{C}$ molar ratio (from sample $\mathrm{B}$ to $\mathrm{H}$ ) are given in Table T2 (see ESI $\dagger$ ). It can be noticed that the colour of the BCNO phosphors can be tuned over nearly entire visible light spectrum. The colour can be tuned by manipulating both the composition ratios of the raw materials as well as the reaction conditions. From these results, we can conclude that the 2D BCNO nanophosphors show promising potential as violet to deep red colour emitting materials because these could be synthesized without using expensive rare earth materials.

The fitting curve of time-resolved photoluminescence decay spectra of as-synthesized powder samples A, B, C, D, E, F, G and $\mathrm{H}$ are shown in Fig. 2c. The PL decay for visible emission at different excitation wavelength for the entire individual sample was recorded. For BCNO nanophosphor, the decay time is in the range of nanoseconds. Since, excitation lifetime is a size dependent parameter, smaller the size of the nanophosphor, the shorter would be the excitation lifetime and higher would be its recombination rate. All the decay curves cannot be fitted by single exponential decay, which implies that multiple processes in addition to the radiative transition may have been largely involved during the decay of emission. The lifetime data of $2 \mathrm{D}$ BCNO nanophosphor transitions are very well fitted to a tripleexponential function as described by the equation:

$$
I(t)=A_{1} \exp \left(-t / \tau_{1}\right)+A_{2} \exp \left(-t / \tau_{2}\right)+A_{3} \exp \left(-t / \tau_{3}\right)
$$

where $\tau_{1}, \tau_{2}$ and $\tau_{3}$ are the decay lifetimes of the luminescence, and $A_{1}, A_{2}$ and $A_{3}$ are the weighting parameters. The observed lifetimes for all the BCNO nanophosphor (samples A, B, C, D, E, F, $\mathrm{G}$ and $\mathrm{H}$ ) and their calculated average lifetime are summarized in Table T3 (see ESI $\dagger$ ). In 2D BCNO nanophosphor, $\mathrm{N}$ vacancy substitutional defects ( $\mathrm{CB}$ or $\mathrm{CN}$ ) and $\mathrm{B} / \mathrm{O}$-related defects are present in the crystalline parts as well as near crystalline sites (in the amorphous parts) and due to this, different defect levels are generated which affects the fluorescent lifetime.

To examine the biocompatibility and cytotoxicity of $2 \mathrm{D}$ BCNO nanophosphor, we used standard MTT assay for B, D, E and $\mathrm{G}$ nanophosphors in human cervical cancer derived HeLa cell line that is one of the well characterized ${ }^{30}$ and most commonly used cancer cell lines in scientific research. Cells with different concentrations of nanophosphors were analyzed including control cells and treatment with established anticancer drug doxorubicin (DOX) or TAXOL as positive control for MTT. As shown in Fig. 3a-d, no apparent cellular toxicity was observed after 48 hour incubation of up to $10 \mu \mathrm{g} \mathrm{mL}$ nanophosphors.

In order to investigate the utility of $\mathrm{B}, \mathrm{D}, \mathrm{E}$ and $\mathrm{G}$ nanophosphors for cellular imaging, we performed in vitro bioimaging studies in HeLa cells using confocal microscopy (Fig. 3e(i), and movie SMV1-3). Fig. 3e(ii) shows the confocal microscopy images of HeLa cells after overnight treatment with nanophosphors. For some of the experiments, the cellular nucleus is stained blue with DAPI (Fig. 3e(iii)). As shown in the confocal images in Fig. 3e(iii), the nanophosphor molecules are distributed throughout the cytoplasm. The overlap of fluorescence and phase contrast images clearly show the cell-specific cellular localization of nanophosphors. The cellular localization of nanophosphors was further confirmed by multi cell imaging for B, D, E and G nanophosphors as shown in Fig. S15 (see ESI $\dagger$ ). The process of blue fluorescent B, red fluorescent $\mathrm{G}$ of 2D BCNO nanophosphor samples and negative control (without BCNO nanophosphor) with DAPI and nucleus can also be clearly seen in ESI Movie $\dagger$ SMV1, SMV2 and SMV3, respectively. The confocal images of HeLa cells with DAPI nuclear staining but without nanophosphors shown in Fig. S16 (see $\mathrm{ESI} \dagger$ ). The images were photographed under similar microscopic conditions used for nanophosphor G. Further, to assess the tissue distribution and fluorescence ability in vivo, BCNO red emitting (sample $\mathrm{G}$ ) and blue emitting (sample $\mathrm{B}$ ) nanophosphors (40 $\mathrm{mg} \mathrm{kg}^{-1}$ body weight) were injected intraperitoneally in six weeks old $\mathrm{C} 57 \mathrm{BL} / 6 \mathrm{~J}$ mice and fluorescence intensity was visualized at different time intervals i.e. $15 \mathrm{~min}$ 

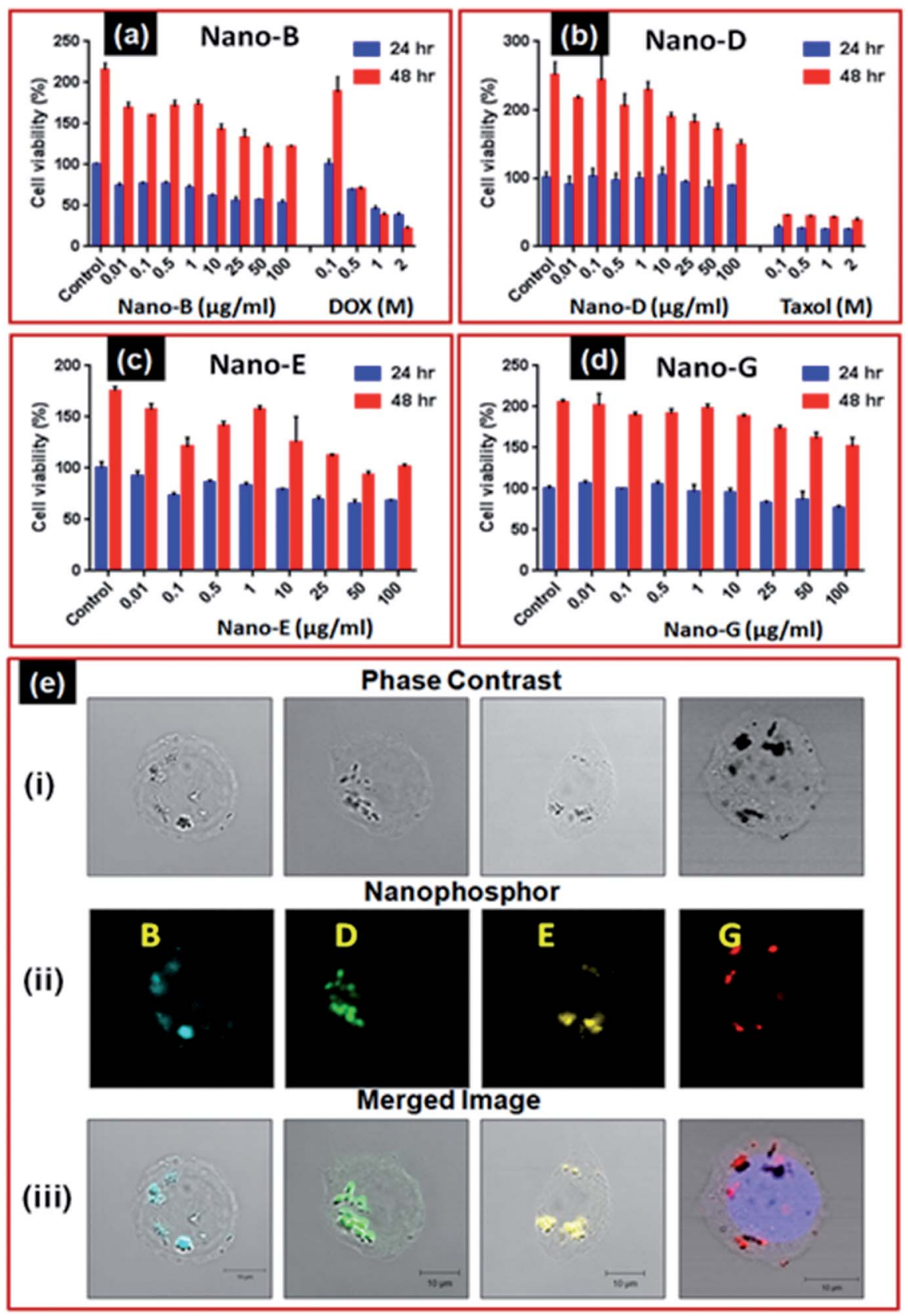

Fig. 3 Cytotoxicity and cell imaging analysis: cell viability assay with human cervical cancer cell line HeLa incubated with as-synthesized (a) sample B, (b) sample D, (c) sample E, and (d) sample G. Established chemotherapeutic drugs DOX and Taxol were used as positive controls for MTT assay, (e) in vitro confocal microscopy images of HeLa cells incubated with 2D BCNO nanophosphor $\left(25 \mu \mathrm{g} \mathrm{mL} \mathrm{L}^{-1}\right)$ for overnight. Sequential images (i) phase contrast of HeLa cells with sample B, D, E and G, (ii) blue fluorescence by sample B, green fluorescence by sample D, yellow fluorescence by sample $E$ and red fluorescence by sample $G$ and (iii) overlap of phase contrast and blue fluorescence, overlap of phase contrast and green fluorescence, overlap of phase contrast and yellow fluorescence and overlap of phase contrast and red fluorescence with blue DAPI nucleus staining, respectively. Scale bars: $10 \mu \mathrm{m}$.

(sample G), $1 \mathrm{~h}$ (sample G) and $2 \mathrm{~h}$ (sample B). 2D BCNO red emitting (sample G) nanophosphors injected mice showed higher fluorescence at $\sim 600 \mathrm{~nm}$ range and fluorescence was seen in the limbs, liver and tail. 2D BCNO blue emitting (sample B) nanophosphors injected mice showed higher fluorescence at $\sim 455 \mathrm{~nm}$ range and fluorescence was seen in the limbs, liver and tail. The maximum intensity was found after $1 \mathrm{~h}$ of injection which gradually declines and negligible after $2 \mathrm{~h}$ shown in
Fig. 4. These experimental mice did not show any loss in weight and exhibited normal activity when compared to control animals. In addition, compounds did not cause any adverse effect to the animals as there was no lethality found.

Thus, the highly luminescent high-contrast imaging capability of nanophosphors strongly suggests that this ultrafine, biocompatible 2D BCNO nanophosphor could be an ideal choice for in vitro as well as in vivo bioimaging applications. 

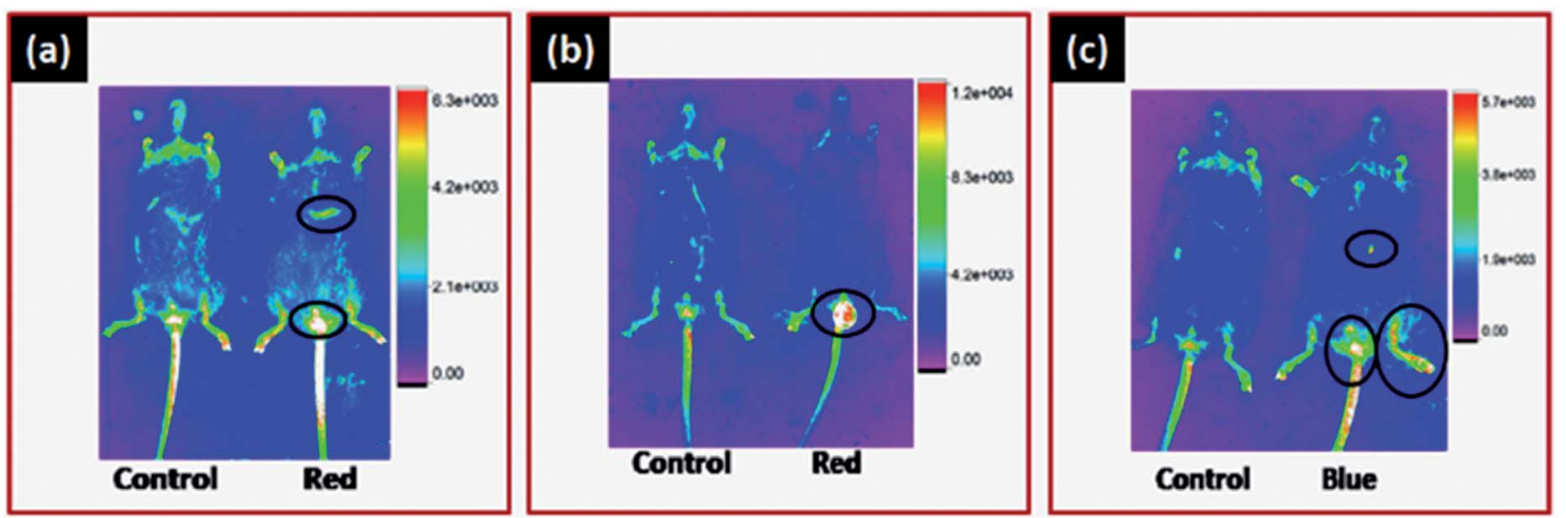

Fig. 4 In vivo imaging in mice using 2D red and blue emitting BCNO nanophosphors (sample G and sample B). Six weeks old, C57BL/6J mice were anesthetized and injected with vehicle and $2 \mathrm{D} B C N O$ red and blue nanophosphors intraperitoneally. Fluorescence images of whole body along with their controls were acquired by exciting at $410 \mathrm{~nm}$ and emission was monitored at $\sim 600 \mathrm{~nm}$ (red) and $\sim 455 \mathrm{~nm}$ (blue). Maximum intensity was observed at $60 \mathrm{~min}$ ( $\mathrm{a}$ and $\mathrm{c}$ ) and gradually declines and negligible after $2 \mathrm{~h} \mathrm{(b).}$

\section{Conclusions}

In summary, simple and inexpensive rare-earth free 2D BCNO nanophosphors of varying compositions have been successfully synthesized using auto-combustion method which can be produce in large-scale. The observed AFM, EELS and TEM/ HRTEM results confirms the formation of $2 \mathrm{D}$ layered $\mathrm{BCNO}$ nanophosphor. The molar ratios of starting materials and heating temperature play a key role in the crystallinity of the material. The absorption peak was narrow and located at 295 nm and with increasing carbon contents, a broad absorption band appeared in the range 300-600 nm. Furthermore, by varying the carbon content in the reaction process, single, distinct, and broad emission band of these 2D BCNO nanophosphors could be achieved, ranging from 342 to $654 \mathrm{~nm}$. These tunable and biocompatible luminescent 2D BCNO nanophosphors are successfully used for in vitro high-contrast cellular imaging as well as in vivo imaging applications. Thus, these novel multi-colour emitting $2 \mathrm{D}$ nanophosphors can act as stepping stone for rare-earth free biocompatible 2D nanoprobes for next generation high-contrast in vitro as well as in vivo imaging applications.

\section{Conflicts of interest}

There are no conflicts to declare.

\section{Acknowledgements}

The authors wish to thank Dr D. K. Aswal (Director, N. P. L.), New Delhi and Prof. O. N. Srivastava (Banaras Hindu University, Varanasi) for their support. The authors gratefully acknowledged University Grant Commission (UGC) and Council of Scientific and Industrial Research (CSIR), Govt. of India for financial assistance to carry out this work. B. A. K. acknowledges the financial support from Dan L. Duncan Cancer Center (DLDCC) and NIH R21 CA17920.

\section{References}

1 A. K. Geim and K. S. Novoselov, Nat. Mater., 2007, 6, 183-191. 2 A. A. Balandin, Nat. Mater., 2011, 10, 569-581.

3 A. H. C. Neto, F. Guinea, N. M. R. Peres, K. S. Novoselov and A. K. Geim, Rev. Mod. Phys., 2009, 81, 109-162.

4 J. Dwivedi, P. Kumar, G. Kedawat and B. K. Gupta, New J. Chem., 2015, 39, 5161-5170.

5 K. Watanabe, T. Taniguchi, T. Niiyama, K. Miya and M. Taniguchi, Nat. Photonics, 2009, 3, 591-594.

6 Y. Kubota, K. Watanabe, O. Tsuda and T. Taniguchi, Science, 2007, 317, 932-934.

7 L. Song, Z. Liu, A. L. M. Reddy, T. N. Narayanan, J. TahaTijerina, J. Peng, G. Gao, J. Lou, R. Vajtai and P. M. Ajayan, Adv. Mater., 2012, 24, 4878-4895.

8 M. S. C. Mazzoni, R. W. Nunes, S. Azevedo and H. Chacham, Phys. Rev. B: Condens. Matter Mater. Phys., 2006, 73, 073108.

9 M. O. Watanabe, S. Itoh, T. Sasaki and K. Mizushima, Phys. Rev. Lett., 1996, 77, 187.

10 X. D. Bai, E. G. Wang, J. Yu and H. Yang, Appl. Phys. Lett., 2000, 77, 67.

11 W. N. Wang, T. Ogi, Y. Kaihatsu, F. Iskandarc and K. Okuyama, J. Mater. Chem., 2011, 21, 5183-5189.

12 W. Lee, D. Portehault, R. Dimova and M. Antoneitti, J. Am. Chem. Soc., 2011, 133, 7121-7127.

13 D. Portehault, C. Giordano, C. Gervias, I. Senkovska and S. Kaskel, Adv. Funct. Mater., 2010, 20, 1827-1833.

14 T. Ogi, Y. Kaihatsu, F. Iskandar, W.-N. Wang and K. Okuyama, Adv. Mater., 2008, 20, 3235-3238.

15 L. Liao, K. Liu, W. Wang, X. Bai, E. Wang, Y. Liu, J. Li and C. Liu, J. Am. Chem. Soc., 2007, 129, 9562-9563.

16 J. Yu and E. G. Wang, Appl. Phys. Lett., 1999, 74, 2948.

17 S. Y. Wang, L. Zhang, Z. Xia, A. Roy, D. W. Chang, J. B. Baek and L. Dai, Angew. Chem., Int. Ed., 2012, 51, 4209-4212.

18 B. K. Gupta, T. N. Narayanan, S. A. Vithayathil, Y. Lee, S. Koshy, A. L. M. Reddy, A. Saha, V. Shanker, V. N. Singh, 
B. A. Kaipparettu, A. A. Martí and P. M. Ajayan, Small, 2012, 8, 3028-3034.

19 B. K. Gupta, V. Rathee, T. N. Narayanan, P. Thanikaivelan, A. Saha, S. P. Singh, V. Shanker, A. A. Marti and P. M. Ajayan, Small, 2011, 7, 1767-1773.

20 H. Konno, T. Ito, M. Ushiro, K. Fushimi and K. J. Azumi, Power Sources, 2010, 195, 1739-1746.

21 X. F. Liu, S. Ye, Y. B. Qiao, G. P. Dong, Q. Zhang and J. R. Qiu, Chem. Commun., 2009, 27, 4073-4075.

22 W. W. Lei, D. Portehault, R. Dimova and M. J. Antonietti, J. Am. Chem. Soc., 2011, 133, 7121-7127.

23 Y. Kaihatsu, W. N. Wang, K. Okuyama, T. Ogi and K. J. Okuyama, J. Electrochem. Soc., 2010, 157, J329-J333.

24 T. Ogi, H. Iwasaki, A. B. D. Nandiyanto, F. Iskandar, W. N. X. Wang and K. Okuyama, J. Mater. Chem. C, 2014, 24, 297-4303.

25 L. Qin, J. Yu, S. Kuang, C. Xiao and X. Bai, Nanoscale, 2012, 4, 120-123.
26 G. Kedawat, S. Srivastava, V. K. Jain, P. Kumar, V. Kataria, Y. Agrawal, B. K. Gupta and Y. K. Vijay, ACS Appl. Mater. Interfaces, 2013, 5, 4872-4877.

27 J. H. Park, S. Vithayathil, S. Kumar, P. L. Sung, L. E. Dobrolecki, V. Putluri, V. B. Bhat, S. K. Bhowmik, V. Gupta, K. Arora, D. Wu, E. Tsouko, Y. Zhang, S. Maity, T. R. Donti, B. H. Graham, D. E. Frigo, C. Coarfa, P. Yotnda, N. Putluri, A. Sreekumar, M. T. Lewis, C. H. Creighton, L. J. C. Wong and B. K. Kaipparettu, Cell Rep., 2016, 14, 2154-2165.

28 V. Brozek and M. J. Hubacek, Solid State Chem., 1992, 100, 120-129.

29 X. Zhang, Z. Lu, J. Lin, L. Li, Y. Fan, L. Hu, X. Xu, F. Meng and J. Tang Zhao, Mater. Lett., 2013, 94, 72-75.

30 R. P. Verma and C. Hansch, Curr. Med. Chem., 2006, 13, 423448. 